\title{
Dual Band Dual Polarized Slot Cut modified Circular Microstrip Antenna
}

\author{
Amit A. Deshmukh \\ EXTC, DJSCOE \\ Vile - Parle (W), \\ Mumbai, India
}

\author{
Sagar Makwana \\ EXTC, DJSCOE \\ Vile - Parle (W), \\ Mumbai, India
}

\author{
Vijay Jain \\ EXTC, DJSCOE \\ Vile - Parle (W), \\ Mumbai, India
}

\begin{abstract}
A new broadband and dual band circular slot cut modified circular microstrip antenna is analyzed and proposed. The parametric study to analyze the effects of circular slot which realizes broader bandwidth is presented. The circular slot creates two additional resonant modes near the fundamental $\mathrm{TM}_{11}$ mode resonance frequency of the equivalent circular patch and yields bandwidth of $202 \mathrm{MHz}$ (8\%). The polarization of the two modes which realizes broadband response was found to be orthogonal, which leads to the principal plane variations over the bandwidth. Further by using this concept, a circular slot cut dual band and dual polarized circular slot cut circular microstrip antenna in 1000 $\mathrm{MHz}$ frequency band is proposed. The proposed antenna yields dual frequency response with orthogonal polarization and variable frequency ratio with bandwidth of $2 \%$ at the dual frequencies.
\end{abstract}

\section{Keywords}

Circular Microstrip Antenna, Broadband microstrip antenna, Dual band microstrip antenna, Dual polarization, Circular slot, Higher order mode

\section{INTRODUCTION}

More commonly broadband and dual band microstrip antenna (MSA) is realized by cutting the slot of different shapes like, U-slot, V-slot, rectangular and toothbrush shaped slot at an appropriate position on the edges or inside the patch [1 -9]. The slot is said to introduce a resonant mode near the fundamental mode resonance frequency of the patch when its length either equals half wave or quarter wave in length. In recent study on slot cut MSAs, it was observed that slot does not introduce any additional mode but reduces the resonance frequency of higher order orthogonal mode of the patch and along with fundamental patch mode yields broadband or dual band response [10 - 12]. In these slot cut MSAs, a single polarization is realized over the complete bandwidth (BW) or at the dual frequencies when the polarization of radiated field due to patch mode and the mode introduced by slot, are in the same direction. The dual polarization is realized when the field due to two modes are orthogonal to each other. More commonly dual polarized MSAs are realized by cutting the slot in the patch center and further by feeding it along the diagonal axis [1]. By selecting appropriate ratio of patch and slot dimensions, a variable frequency ratio has been realized [1]. With respect to the fundamental mode of operation, compact MSA is realized by cutting the slot inside the patch or by placing the shorting post along the zero field line in the patch [13]. The slot lengthens the surface current length and reduces its resonance frequency for given patch size whereas shorting technique converts half wave length resonator into a quarter wavelength resonator. The conventional half wave length resonator shows broadside radiation pattern with lower cross polarization levels. Due to an un-symmetrical field distribution around the patch edges, shorted and slot cut
MSAs shows radiation pattern with higher cross polar levels. In application like mobile communication, since the polarization of incoming wave is not known, MSAs with higher cross polar levels will be useful. Recently a new circular slot cut circular MSA (CMSA) and key shaped slot cut CMSA is reported [14]. The equivalent CMSA operates at frequency of $2520 \mathrm{MHz}$ and $5120 \mathrm{MHz}$ which corresponds to the $\mathrm{TM}_{11}$ and $\mathrm{TM}_{02}$ modes of circular patch. At each of the above mode, CMSA gives lower BW of 1 to $2 \%$. To enhance the BW, a circular slot was cut inside and towards the periphery of the patch and another smaller radius circular patch was placed inside the slot. This configuration operates at 2470 and $5830 \mathrm{MHz}$ with BW of $3.45 \%$ and $3.03 \%$, respectively at the two frequencies. To improve this $\mathrm{BW}$ at dual frequencies, key shaped slot cut MSA was proposed [14].

In this paper, first an analysis of reported circular slot cut CMSA is presented. The behavior of slot cut CMSA around its fundamental $\mathrm{TM}_{11}$ mode $(2000$ to $3000 \mathrm{MHz}$ ) is studied. Around that frequency range/mode, a broadband response with the formation of loop inside the VSWR $=2$ circle in the smith chart is observed, which shows BW of $202 \mathrm{MHz}(8 \%)$. The radiation pattern over the $\mathrm{BW}$ was found to be in broadside direction. However, a variation in E-plane direction from $\Phi=90^{\circ}$ to $0^{\circ}$, is noticed over the BW. Therefore a detail analysis to study the effect of circular slot in CMSA, is presented. It was observed that, circular slot creates two resonant modes (degenerates fundamental mode into two modes) around the $\mathrm{TM}_{11}$ mode frequency of CMSA. The parametric study for variations in circular slot dimensions and its position is presented. When the circular slot is placed towards the perimeter of the patch the first of dual frequencies reduces whereas second frequency increases thereby realizing smaller frequency ratio of 1.0 to 0.9 . However when the circular slot is placed closer to the center of patch, both the frequencies reduces and realizes variable frequency ratio in the same range. For different slot position, at first frequency, the surface currents maximum is aligned along $\Phi=90^{\circ}$ whereas at second frequency they are aligned along $\Phi=0^{0}$. Therefore at first frequency, E-plane is aligned along $\Phi=90^{\circ}$, whereas at second frequency it is aligned along $\Phi=0^{0}$. Thus the slot cut CMSA will realize dual band dual polarized or broadband dual polarized response. Further in $1000 \mathrm{MHz}$ frequency band and using glass epoxy substrate $\left(\varepsilon_{\mathrm{r}}=4.3, \mathrm{~h}=\right.$ $0.16 \mathrm{~cm}, \tan \delta=0.02)$, a dual band dual polarized design of circular slot cut CMSA is proposed. With respect to the slot position the proposed MSA gives dual band and dual polarized response with $2 \% \mathrm{BW}$ at the individual frequencies. The analysis of the circular slot cut CMSA is first carried out using IE3D software [15]. For measurements, the patch in $1000 \mathrm{MHz}$ frequency band was fabricated and it was fed using SMA panel type connector of $0.12 \mathrm{~cm}$ inner wire diameter. The measurement was carried out using $\mathrm{R} \& \mathrm{~S}$ vector network analyzer. As the circular slot cut CMSA was investigated on low cost lossy substrate they have gain less than $0 \mathrm{dBi}$. To 
improve upon the gain, suspended configurations of slot cut CMSAs can be used, which will also increase the BW. The radiation pattern was measured in minimum reflection surroundings with required minimum far field distance between reference antenna and antenna under test [16].

\section{CIRCULAR SLOT CUT CMSA}

The reported circular slot cut CMSA is shown in Fig. 1(a, b). The patch is fabricated on FR4 substrate having parameters, $\varepsilon_{\mathrm{r}}$ $=4.4, \mathrm{~h}=0.159 \mathrm{~cm}, \tan \delta=0.025$. For CMSA, measured resonant frequencies for observed $\mathrm{TM}_{11}$ and $\mathrm{TM}_{02}$ modes are, 2520 and $5120 \mathrm{MHz}$, respectively as shown in Fig. 1(c) [14]. Since the BW at dual frequencies is smaller, an offset circular slot is cut inside the patch, as shown in Fig. 1(a, b) [14]. The radius of the inner and outer circle is, 0.2 and $0.4 \mathrm{~cm}$, respectively. The observed dual frequencies for the slot cut CMSA are, 2470 and $5830 \mathrm{MHz}$, with nearly $3 \% \mathrm{BW}$, at the two frequencies, as shown in Fig. 1(c) [14]. The return loss $\left(\mathrm{S}_{11}\right)$ plot for slot cut CMSA shows proximity of two resonant modes towards the higher frequency range. To analyze the configuration, for $\mathrm{x}_{\mathrm{f}}=0.7$ and $\mathrm{y}_{\mathrm{f}}=0.6 \mathrm{~cm}$, slot cut CMSA is simulated using IE3D software in 2000 to $6000 \mathrm{MHz}$ frequency range and its resonance curve plot is shown in Fig. 1(d). The resonance curve shows proximity of two resonant modes around $2500 \mathrm{MHz}$ and $5500 \mathrm{MHz}$. These frequencies are closer to $\mathrm{TM}_{11}$ and $\mathrm{TM}_{02}$ mode resonance frequencies of the equivalent CMSA. To study the response of slot cut CMSA around its $\mathrm{TM}_{11}$ mode frequency, the MSA is simulated in the frequency range of 2000 to $3000 \mathrm{MHz}$ and its input impedance plot is shown in Fig. 1(e). The plot shows the formation of loop inside the VSWR $=2$ circle, indicating the broadband response. The realized BW is from 2407 to 2607 $\mathrm{MHz}(202 \mathrm{MHz}(8 \%))$. This broader BW is realized due to the two adjacent modes present near the $\mathrm{TM}_{11}$ mode frequency of equivalent CMSA as observed in the resonance curve plot. The surface current distributions at the two modes (peaks) are shown in Fig. 2(a, b). The distributions at both the frequencies are similar to the $\mathrm{TM}_{11}$ mode of CMSA and they show nearly half wavelength variations along half of the patch perimeter. However at first frequency, maximum of surface currents is aligned along $\Phi=90^{\circ}$ whereas it is along $\Phi=0^{0}$, at second frequency. This leads to the variation in E-plane direction from $\Phi=90^{\circ}$ to $0^{\circ}$, over the VSWR BW. The surface current contribution on the inner smaller circular patch was found to be minimum. Since radius of smaller circular patch is smaller, its resonance frequency does not lie near the resonance frequency of $\mathrm{TM}_{11}$ mode of CMSA. Therefore without the inner circular patch, slot cut CMSA shows similar result in 2000 to $3000 \mathrm{MHz}$ frequency range, to that shown in Fig. $1(\mathrm{e})$.

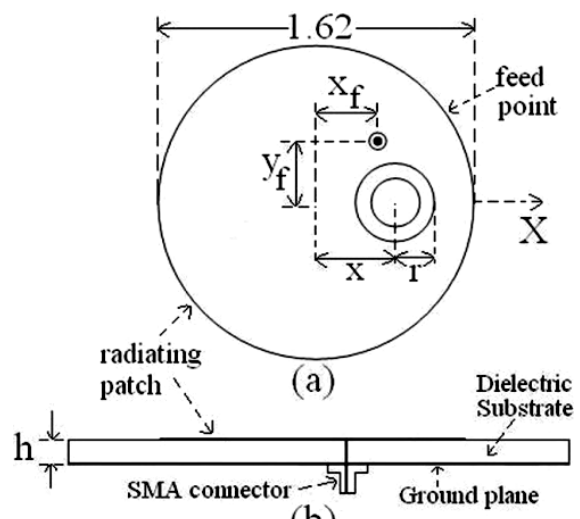

(b)
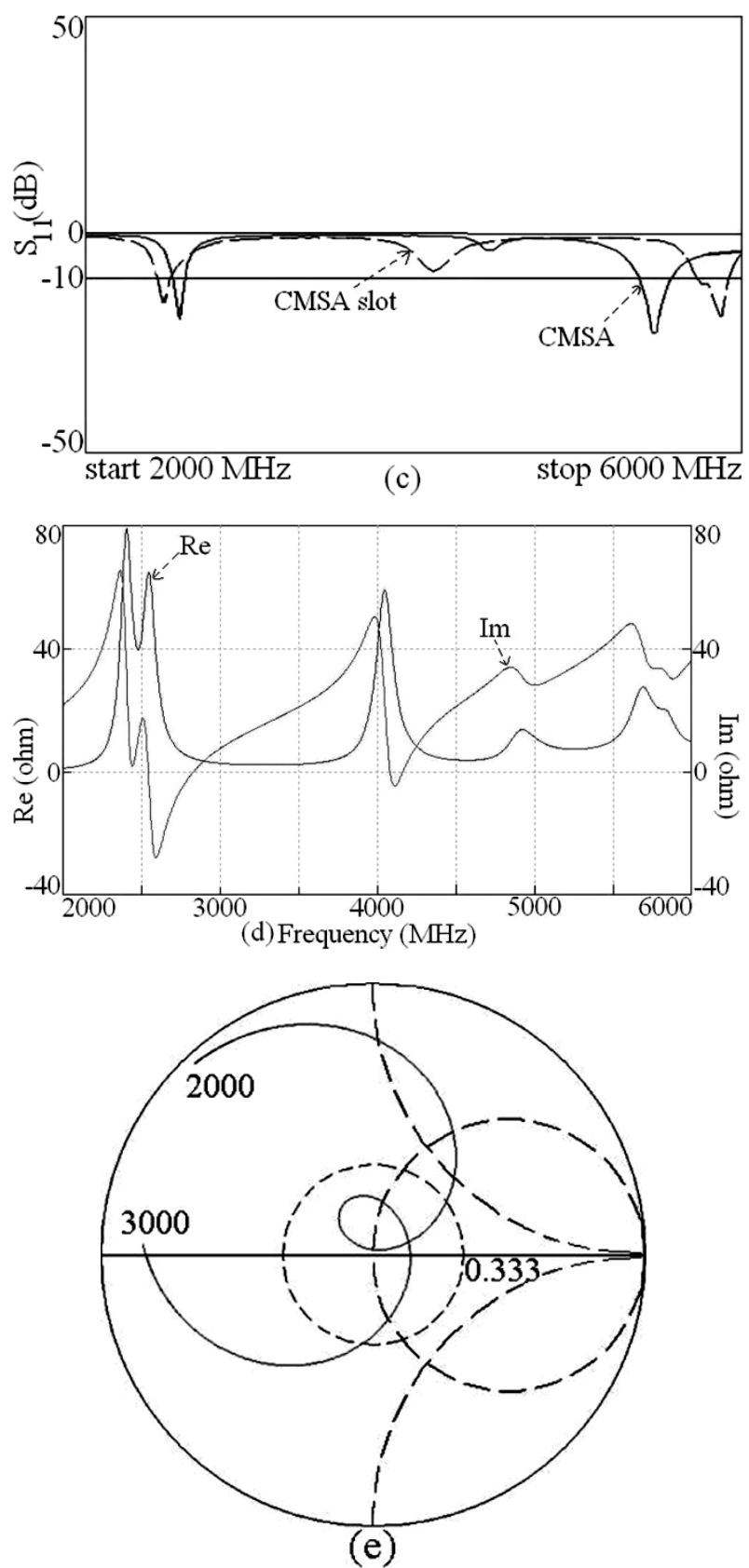

Fig. 1 (a) Top and (b) side views, (c) measured return loss plots [14], simulated (d) resonance curve and (e) input impedance plots for circular slot cut CMSA

To analyze the configuration for the excitation of two orthogonal modes, parametric study is carried out. For $\mathrm{x}=1.0$ $\mathrm{cm}$ and $\mathrm{r}=0.5 \mathrm{~cm}$, slot cut CMSA is simulated for different feed point locations, as shown in Fig. 2(c) and there resonance curve plots in 2000 to $3000 \mathrm{MHz}$ frequency range are shown in Fig. 2(d). 


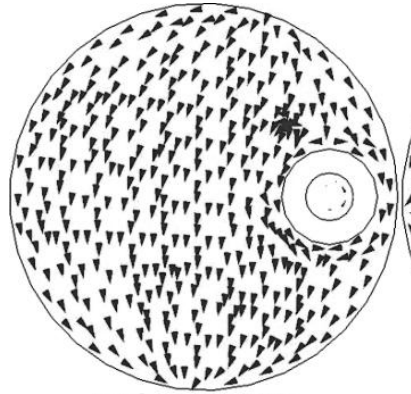

(a) $\mathrm{f}_{1}=2409 \mathrm{MHz}$

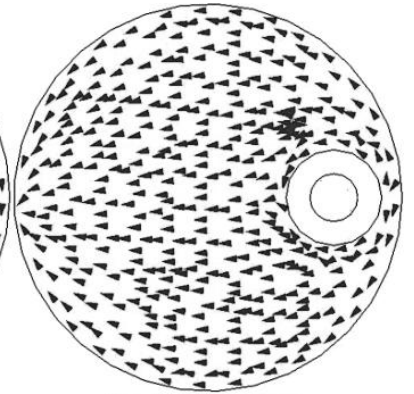

(b) $\mathrm{f}_{2}=2557 \mathrm{MHz}$

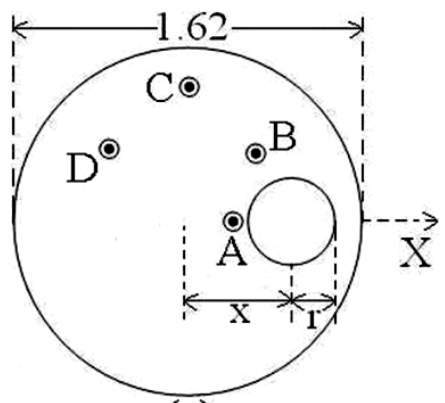

(c)

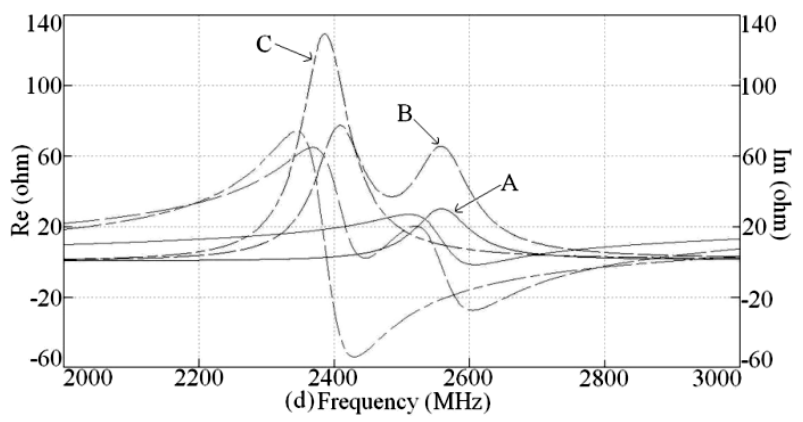

Fig. 2 (a, b) Surface current distribution at dual frequencies, (c) circular slot cut CMSA and its (d) resonance curve plots for different feed point locations

When the feed point is located either along $\mathrm{x}$ or $\mathrm{y}$-axis i.e. at feed point location ' $\mathrm{A}$ ' or ' $\mathrm{C}$ ', a single mode is observed in the resonance curve plot since the feed point is located at the minimum impedance location of the corresponding orthogonal mode. Further the effects of variations in circular slot radius and its position on the dual frequencies are studied and they are plotted in Fig. 3(a, b). The surface current distributions at second frequency for $\mathrm{r}=0.4 \mathrm{~cm}$ and $\mathrm{x}=0.5$ and $1.0 \mathrm{~cm}$, are shown in Fig. 3(c, d). Also the resonance curve plots for varying slot position and slot radius for given feed point location are shown in Fig. 4(a, b). For smaller slot radius ( $\mathrm{r} \leq$ $0.4 \mathrm{~cm})$, when circular slot is placed near the patch centre $(\mathrm{x} \leq$ $0.5 \mathrm{~cm}$ ), two distinct modes are not observed in the resonance curve plot. This is because smaller slot radius does not create an orthogonal unequal path lengths or degenerates $\mathrm{TM}_{11}$ mode into two orthogonal modes. For smaller slot radius, this is observed when the slot is placed more towards patch edge as it increases un-symmetry in the structure. When the slot is placed close to the patch centre, it increases surface current lengths at both the modes which reduce their frequencies. However when the slot is placed close to the patch edge, first of the dual frequency reduces whereas second frequency increases. As observed from the surface current distributions at second mode, when the slot is placed more towards the patch edge, surface currents does not start from the patch edge, which reduces the effective patch radius, that increases its resonance frequency. The first frequency reduces due to an increase in surface current lengths due to the slot. For both the values of slot position, variable frequency ratio in the range of 1 to 0.9 is observed. As observed from Fig. 4(a, b), when the slot radius is higher, both the frequencies reduce. Whereas when slot position is changed (i.e. from $\mathrm{x}=0.5$ to $1.0 \mathrm{~cm}$ ), for given slot radius, first frequency remains nearly constant whereas second frequency increases.

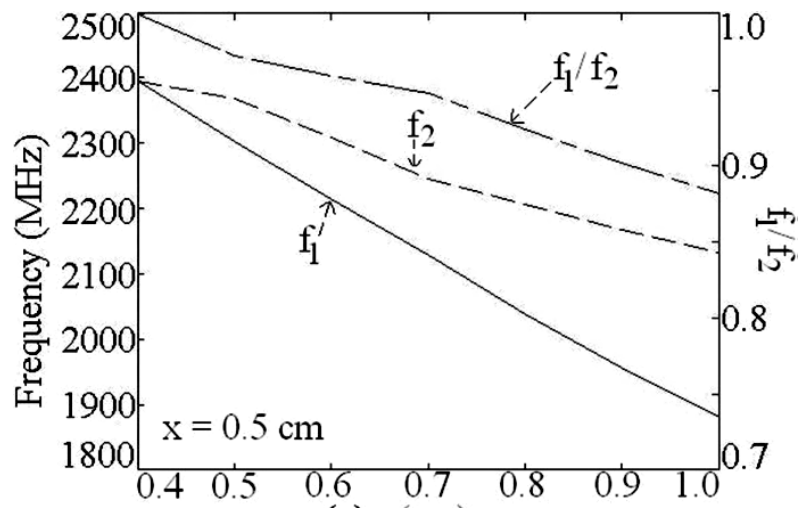

(a) $\mathrm{r}(\mathrm{cm})$
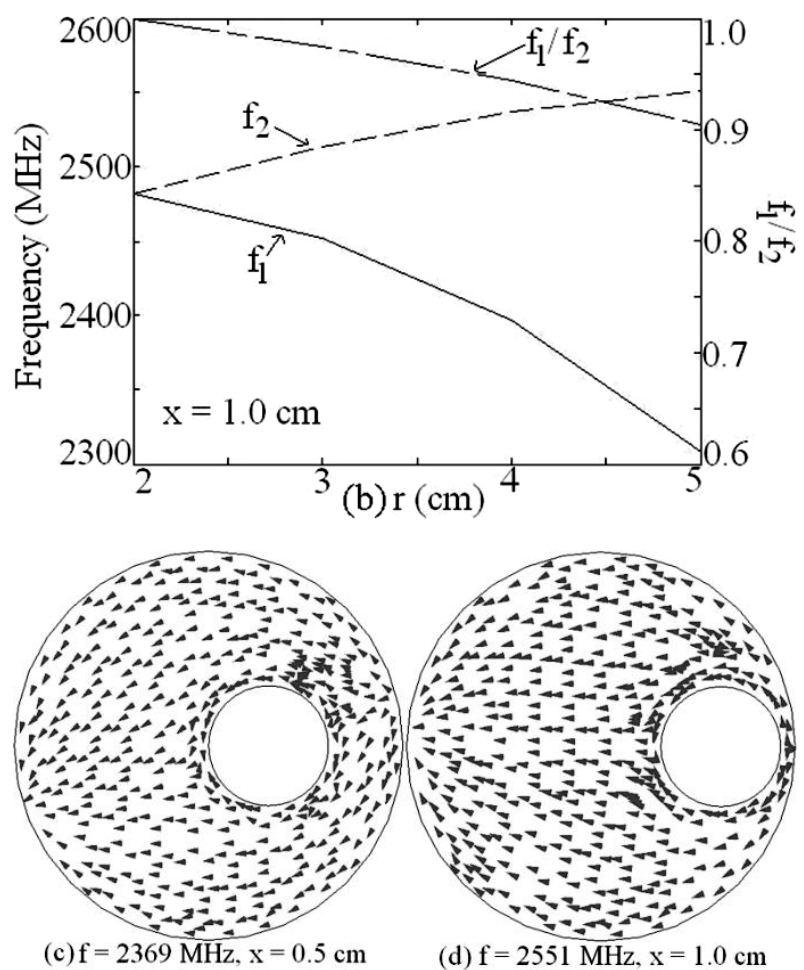

Fig. 3 (a, b) Variation in dual frequencies and their ratio for different circular slot positions and radius, and (c, d) surface current distributions at second frequency for circular slot cut CMSA

The optimum dual band response in above slot cut CMSA is realized by controlling the coupling between the two modes. This is realized for circular slot placed more towards the patch edges and by placing the feed point on the opposite side (point 'D') as shown in Fig. 2(c). For $x=1.0 \mathrm{~cm}$ and $r=0.5 \mathrm{~cm}$, the optimized simulated and measured input impedance plots is shown in Fig. 4(c). The simulated dual frequencies and BW's are, 2322 and $2563 \mathrm{MHz}$ and 72 and $90 \mathrm{MHz}$, respectively. 
The measured dual frequencies and BW's are, 2345 and 2601 $\mathrm{MHz}$ and 78 and $102 \mathrm{MHz}$, respectively.
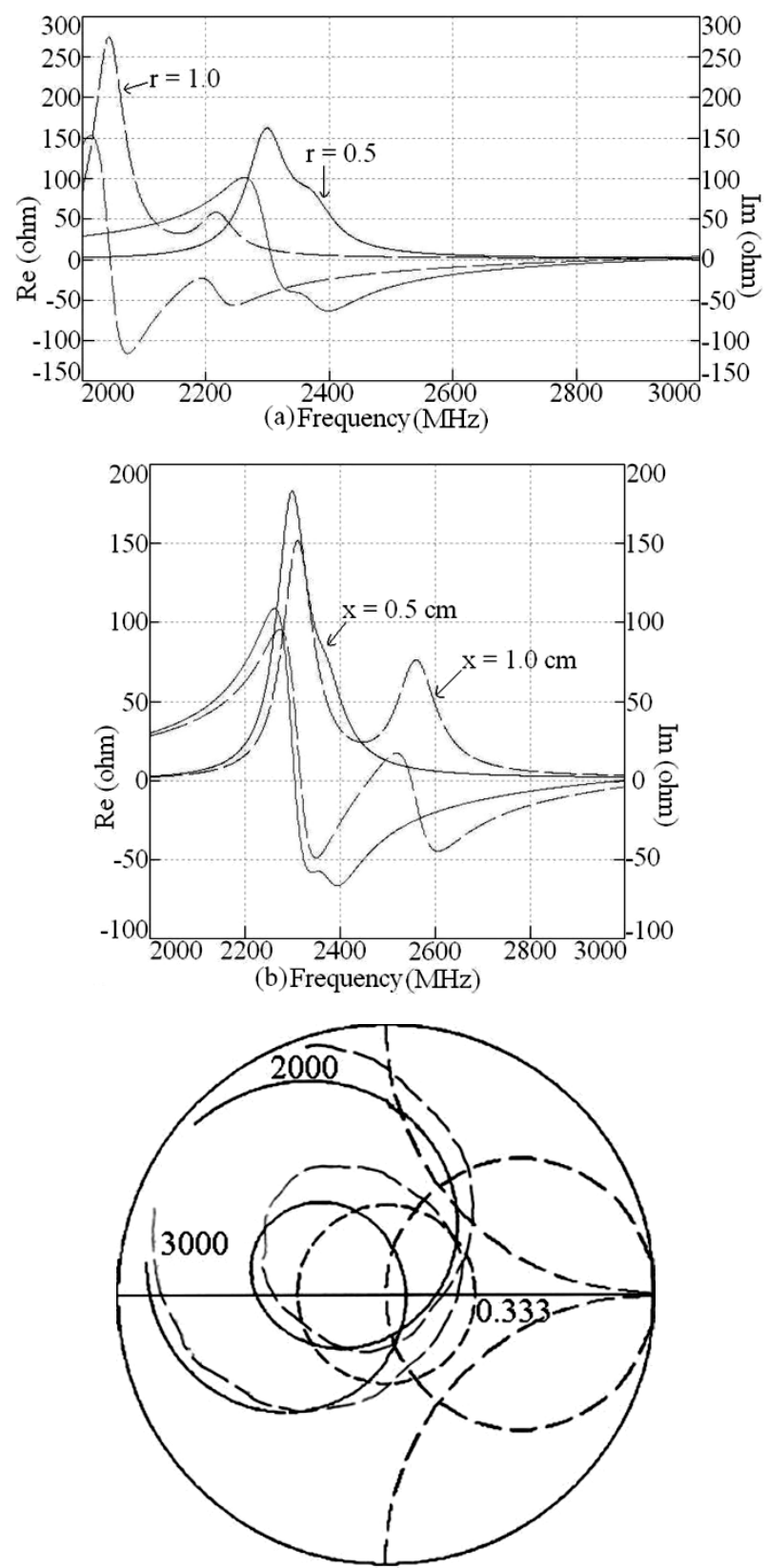

(c)

Fig. 4 Resonance curve plots for (a) variations in slot radius, $x=0.5 \mathrm{~cm}$ and (b) position, $r=0.5 \mathrm{~cm}$ and (c) optimized dual band response, $(-)$ simulated, $(--)$ measured for slot cut CMSA

Further by using the above concept, dual band and dual polarized circular slot cut CMSA is designed in $1000 \mathrm{MHz}$ frequency band. Using glass epoxy substrate $\left(\varepsilon_{\mathrm{r}}=4.3, \mathrm{~h}=\right.$ $0.16 \mathrm{~cm}, \tan \delta=0.02)$ the patch radius for $\mathrm{TM}_{11}$ mode frequency to be around $1000 \mathrm{MHz}$, is calculated to be $4.5 \mathrm{~cm}$. The slot is cut inside the patch and for different values of slot position (x) and its radius (r), the variation in dual frequency is shown in Fig. 5(a, b). The similar behavior to that observed in above reported slot cut CMSA is present. Further for $\mathrm{x}=$ $3.0 \mathrm{~cm}$ and $\mathrm{r}=1.4 \mathrm{~cm}$, the optimized dual band and dual polarized response is realized as shown in Fig. 5(c).

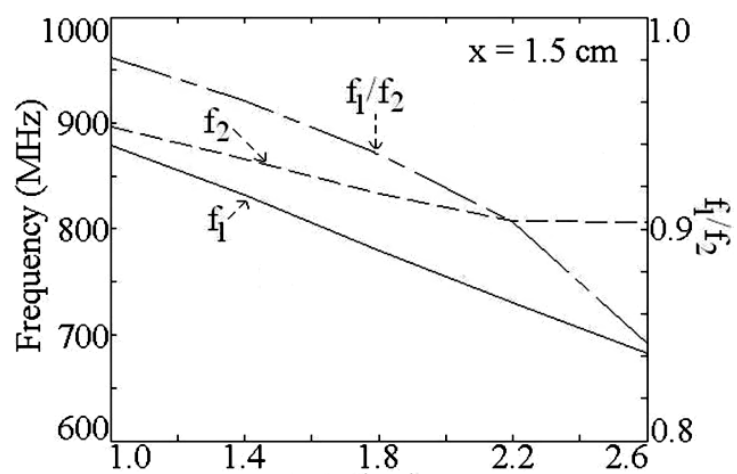

(a) $\mathrm{r}(\mathrm{cm})$

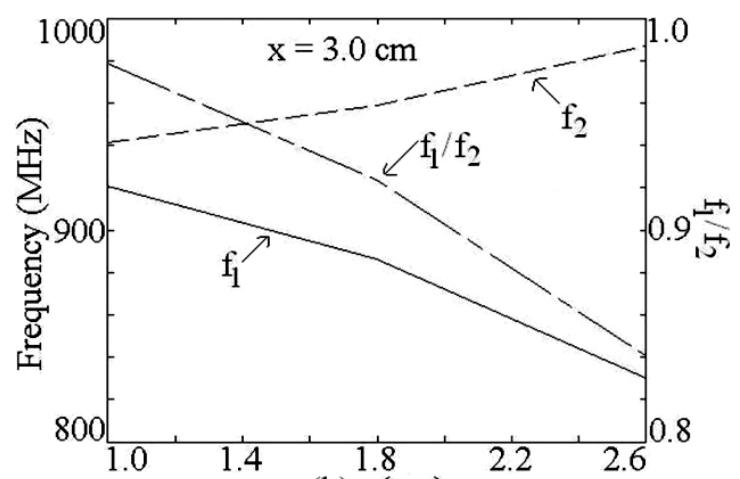

(b) $\mathrm{r}(\mathrm{cm})$

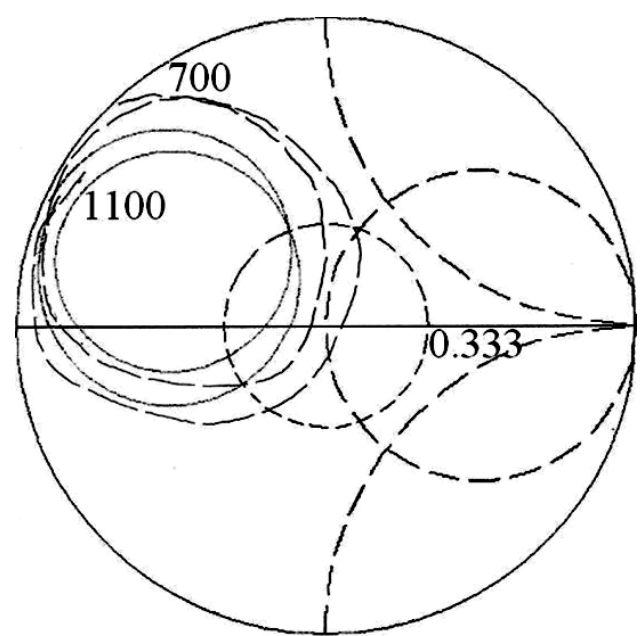

(c)

Fig. 5 (a, b) Variation in dual frequency and their ratio for different circular slot positions and radius and (c) optimized dual band response for slot cut CMSA in 1000 MHz frequency band, $(-)$ simulated, $(--)$ measured

The simulated dual frequencies and BW's are, 825 and 980 $\mathrm{MHz}$ and 12 and $18 \mathrm{MHz}$, respectively. The patch was fabricated using glass epoxy substrate of dimension $20 \mathrm{~cm}$ side length and the experiment was carried out using R \& S vector network analyzer. The measured dual frequencies and BW is 836 and $975 \mathrm{MHz}$ and 11 and $19 \mathrm{MHz}$, respectively as shown in Fig. 5(c). The fabricated prototype of the configuration is shown in Fig. 6(a). The radiation pattern at dual frequencies is shown in Fig. $6(\mathrm{~b}, \mathrm{c})$. The pattern is in the broadside direction with E-plane aligned along $\Phi=90^{\circ}$ at first frequency and along $\Phi=0^{\circ}$ at second frequency. Since the size of the ground plane is taken to be larger than patch size, the back-lobe radiation is smaller and hence not shown in the radiation pattern plots. 


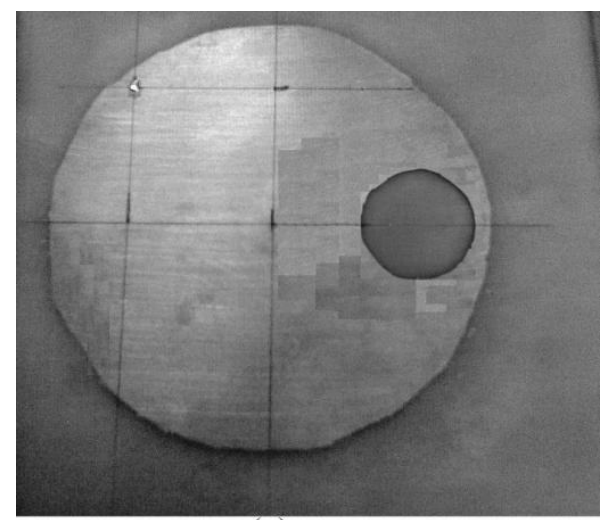

(a)

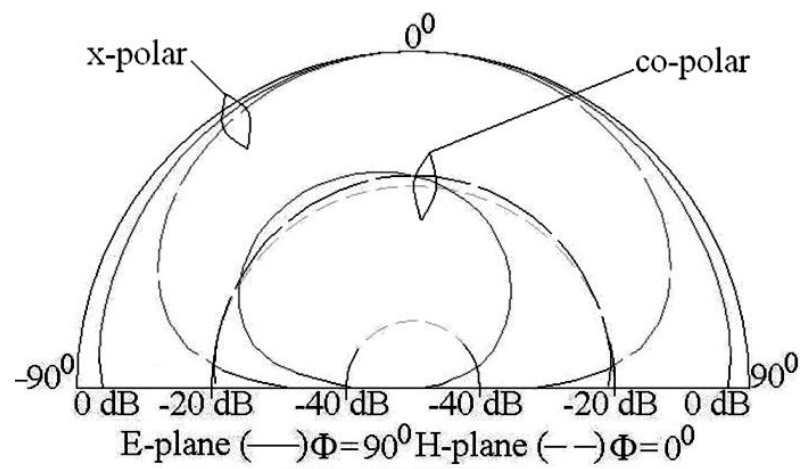

(b) $\mathrm{f}=836 \mathrm{MHz}$

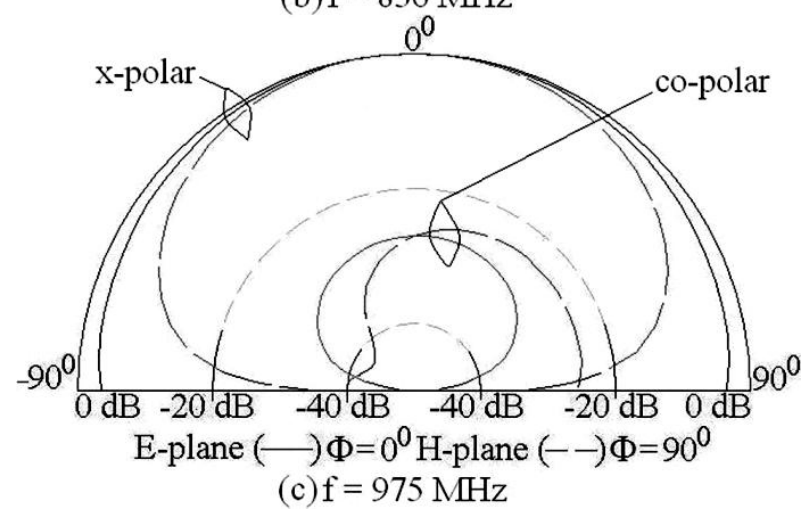

Fig. 6 (a) Fabricated prototype and (b, c) radiation pattern at dual frequencies for dual band circular slot cut CMSA

\section{CONSLUSIONS}

The dual band and dual polarized circular slot cut CMSA in $2000-3000 \mathrm{MHz}$ frequency band is analyzed and proposed. In circular slot cut CMSA, for larger slot radius or when slot is placed more towards the CMSA edges, slot degenerates $\mathrm{TM}_{11}$ mode of CMSA into two orthogonal modes. Further for different slot radius and their positions it realizes dual band dual polarized response with variable frequency ratio in the range of 1 to 0.9 . The slot cut dual band dual polarized CMSA in $1000 \mathrm{MHz}$ frequency band is proposed. The proposed CMSA yields nearly $2 \% \mathrm{BW}$ at the dual frequencies with variable frequency ratio of 1 to 0.8 .

\section{REFERENCES}

[1] Wong, K. L. 2002. Compact and Broadband Microstrip Antennas, John Wiley \& sons, Inc., New York, USA
[2] Huynh, T., and Lee, K. F. 1995. Single-Layer SinglePatch Wideband Microstrip Antenna, Electronics Letters, vol. 31, no. 16, (August 1995), 1310-1312.

[3] Guo, Y. X., Luk, K. M., Lee, K. F., and Chow, Y. L. 1998. Double U-slot Rectangular Patch Antenna, Electronics Letters, vol. 34, (1998), 1805 - 1806

[4] Sharma, S. K., and Shafai, L. 2009. Performance of a Novel $\Psi$-Shaped Microstrip Patch Antenna with Wide Bandwidth, IEEE Antennas \& Wireless Propagation Letters, vol. 8, (2009), $468-471$.

[5] Lee, K. F., Yang, S. L. S., Kishk, A. A., and Luk, K. M. 2010. The Versatile U-slot Patch, IEEE Antennas \& Propagation Magazine, vol. 52, no. 1, (February 2010), $71-88$

[6] Wong, K. L., and Hsu, W. H. 2001. A Broadband Rectangular Patch Antenna with a Pair of wide slits, IEEE Transactions on Antennas \& Propagation, Vol. 49, No. 9, (September 2001), 1345 - 1347.

[7] Yang, F., Zhang, X., Yc, X., and Sammi, Y. R. 2001. Wide-band E-shaped patch antennas for wireless communication, IEEE Transactions on Antennas \& Propagation, vol. 49, no. 7, (July 2001), $1094-1100$.

[8] Lee, K. F., Yang, S. L. S., and Kishk, A. A. 2008. Dual and Multi-band U-slot patch Antennas, IEEE Antennas and wireless Propagation Letters, vol. 7, (2008), 645 647.

[9] Vedaprabhu, B., and Vinoy, K. J. 2010. An Integrated Wideband Multifunctional Antenna using a Microstrip patch with two U-slots", Progress In Electromagnetic Research B, vol. 22, (2010), 221 - 235.

[10] Deshmukh, Amit A., Joshi, Apurva A., and Tirodkar, T., Broadband slot cut gap-coupled proximity fed E-shaped Microstrip Antenna, International Journal of Computer Application, vol. 68, no. 17, April 2013, 15 - 18

[11] Deshmukh, A. A., Ray, K. P., and Kadam, A. 2013. Analysis of slot cut Broadband and Dual band Rectangular Microstrip Antennas, IETE Journal of Research, vol. 59, no. 3, (May - June 2013), 193 - 200.

[12] Deshmukh, A. A., and Ray, K. P. 2013. Analysis of Broadband $\Psi$-shaped Microstrip Antennas, IEEE Magazine on Antennas and Propagation, vol. 55, no. 2, (June 2013), 107- 123.

[13] Kumar, G., and Ray, K. P. 2003, Broadband Microstrip Antennas, First Edition, USA, Artech House

[14] Garima, Bhatnagar, D., Saini, J. S., and Saxena, V. K. 2010. Modified circular patch antenna with key shape slot for wireless communication systems, International Journal of Microwave and Optical Technology, vol. 5, no. 6, (November 2010), $396-401$.

[15] IE3D 12.1, 2004. Zeland Software, Freemont, USA

[16] Balanis, C. A., Antenna Theory: analysis and design, $2^{\text {nd }}$ edition, John Wiley \& Sons Ltd. 